\title{
Upcoming: April 16-21, MRS Spring Meeting in San Francisco
}

\begin{abstract}
To list an event in the Calen-
dar, contact J. Dininny,

Materlals Research Society,

9800 MeKnight Road,

Pittsburgh, PA 15237; (412)

367-3036; tax (412) 367-4373.

MIRIS Identifles meetings sponsored or co-sponsored by the Materials Research Society; boldtace type without ine logo identifies MRS-endorsed meetlngs.
\end{abstract}

SEe MAS BULLETIN VoI. XV No. 2 for Calendar events from March 1 through March 31, 1990.

\section{APRIL 1990}

16

8th Intemational Conference on Thin Films, in conjunction with Intemational Conference on

Metallurgical Coatings

San Diego, CA

W.D. Sproul, Bas. Ind. Res. Lab. Northwestem Univ., 1801 Maple

Ave. Evanston, IL 60201-3135;

(312) 491-4108; tax (312)

$491-4486$

17

1st Intemational Conference on Epitaxial Crystal Growth

Budapest, Hungary

E. Lendvay, Res. Inst. for Tech.

Phys., Hungarian Acad. of Sci.

Ujpest 1, Pf 76, Budapest.

Hungary-1325; $\operatorname{tax}(36)$ (1) 698-037

$2-6$

Intemational Congress on Optical

Science and Engineering

Hamburg. W. Germany

SPIE, P.O. Box 10, Bellingham,

WA 98227-0010; (206) 676-3290;

$\operatorname{tax}(206) 647-1445$

$2-6$

Advances in Materials Science and Applications of High-Temperature

Superconductors

Greenbelt, MD

Y. Flom, NASA Goddard Space

Flight Center, Code 313.0, Bldg.

22. Rm. 339, Greentelt, MD

2077 ; (301) 286-3274

3rd Annual Buffalo Symposium on Materials and Processes

Butfalo, NY

C. Lorge, Wilson Greartatch Ltd. 10,000 Wehrte Drive, Clarence, NY 14031; (716) 959-6901

46

Fine Ceramic Powders University of Warwick, United Kingdom

Institute of Ceramics, Shelton

House, Stoke Rd., Shetton,

Stoke-on-Trent ST4 2DR; (0782)

202116; $\operatorname{tax}$ (0782) 202421

\section{7}

Physics of Advanced Materials

Systems

Alfred, NY

E.C. Behrman, New York State

Section of the American Physica

Society, Alfred Univ. Alfred, NY

14802; (607) 871-2249

8-13

American Crystallographic

Association Meeting

New Orleans, LA

ACA, P.0. Box 96, Ellicott Station

Buffalo, NY 14025-0096; (716)

856-9600 ext. 321; tax (716)

$852-4846$

10-12

New Materials and Their

Applications

Warwick, United Kingdom

Institute of Physics, 47 Belgrave

Square, London SW1X 80X

United Kingdom; 01-235 6111;

fax 01-2596002

$12-13$

3rd Intemational Conference on

Amorphous and Crystalline

Silicon Carbide and other Group

IV-IV Materials

Washington, DC

G.L. Harris, Materials Science

Research Ctr. of Excellence

Howard Univ./School of Engineer-

ing, 2300 Sixth St. NW, Washing

ton, DC 20059: (202) 636-6618

$\operatorname{tax}(202) 636-5960$

16-20

Florida Catalysis Conference

Palm Coast, $\mathrm{FL}$

R.S. Drago, Dept. of Chemistry,

Univ. of Florida, Gainesville, FL

32611

\section{6-21}

\section{MIRIS Materlals Researc|}

Society Spring Meeting

San Francisco, CA

M. Geil, Materials Research

Society, 9800 McKnight Rd.,

Pittsburgh, PA 15237; (412) 367

3003; $\operatorname{tax}(412)$ 367-4373

22.26

ACerS 92nd Annual Meeting

Dallas, TX

American Ceramic Society, 757

Brooksedge Plaza Dr., Westerville,

$\mathrm{OH} 43081$; (614) 890-4700; tax (614) $899-6100$

22-27

American Chemical Society

Meeting

Boston, MA

American Chemical Society, 1155

16th St. NW, Washington, DC

20036; (202) 872-4600

23-25

Material Aspects of Machining

Bugge, Belgium

R. Peys, Ingenieurshuis vaw,

Desquintei 214, B-2018 Antwer-

pen; 03/216 09 96;

$\operatorname{tax} 03 / 2160689$
23-25

2nd Intemational Conference on Indium Phosphide and Related Materials

Denver, CO

Lasers and Electro-0ptics Society,

445 Hoes Lane, P.O. Box 133

Piscataway, NJ 08855-1331; (201)

562.3895; $\operatorname{tax}$ (201) 562-1571

23-26

Intemational Conference on Low

Temperature Electronics

Berkeley, CA

M. Vukovojac, Butterworth

Scientific Ltd. P.O. Box 63,

Westbury House, Bury St.

Guildford, Sumey GU2 5BH

United Kingdom; 0483 30096;

tax 0483301563

\section{3-27}

MASHTEC '90: Intemational

Conference on Materials Science

for High Technologies

Dresden, E. Germany

W. Pompe, MASHTEC ' 90

Zentralinstitute tür Festhörperphy-

sik and Werkstoftorschung der

AOW der DDR, Helmholtestr. 20

Dresden, DDR-8027.

East Germany

24

26th Symposium of the New

Mexico Chapter of the American

Vacuum Society

Abuquerque, NM

D. Harradine, Los Alamos

National Laboratory, MS $\mathbf{5 6 6 7}$

Los Alamos, NM 87545

(505) 667-1176

24-27

13th Intemational Cryogenic

Engineering Conterence

Beifing, China

C.S. Hong, Cyogenic Lab.

Academia Sinica, AS, P.0. Box

2711, Beiīing 100080 China; 861-

256-4051; tax 861-256-4049

25-27

Cadcomp'90: Computer Aided

Design in Composite Material

Technology

Brussels, Belgium

M. Bounlau, CADCOMP 90 VuB

TW (KB), Pleinlaan 2, B-1050

Bussels, Belgium; $32(0)$

26412922

25-27

12th Symposium on Applied

Surface Analysis

Albuquerque, NM

D. Hamadine, Los Alamos

National Laboratory MS J567,

Los Alamos, NM 87545;

(505) 667-1176

29-4

Society of Vacuum Coaters 33r

Annual Technical Conference

New Orleans, LA

SVC Administrajue Office, 400

Live Oak Loop. Albuquerque, NM

87122; (505) 298-7624; $\operatorname{tax}(505)$

$298-7942$
294

Intemational Conference on

Transport Properties of Super-

conductors

Ro de Janeiro, Brazl

R. Nicolsky, Instituto de Física,

Universidade Federal do Rio de

Janeiro, C.P. 68528, 21945 Rio de

Janeiro. Brazil; (55) (21) 270-1191/

270-1736; $\operatorname{tax}(55)$ (21) 280-7093

$30-4$

Conterence on the Science and

Technology of Thin Film Super-

conductors

Denver $\mathrm{CO}$

D. Christodaro, Solar Energy

Research Institute, Conf.

Coordination Section, 1617 Cole

Blvd., Golden CO 80401-3393;

(303) 231-1158; fax (303) 231-1199

\section{MAY 1990}

3

Atvanced Materials for the 90s

Washington, DC

TMS, 420 Commonweatth Dr.

Warrendale, PA 15086; (412) 776

9050; $\operatorname{tax}(412) 776-3770$

$6-8$

Symposium on High $T_{G}$ Superconductor Technologies at the 177th Electrochernical Society Meeting

Montreal, Canada

S. Raider, IBM T.J. Watson

Research Ctr. P.0. Box 218 ,

Yorktown Heinhts, NY 10598

(914) $945-2203$

6-11

177th Electrochemical Society

Meeting

Montreal, Canada

Electrochemical Society $10 \mathrm{~S}$

Main St., Pennington, NJ 08534

2896; (609) 737-1902

911

ICMC-90: High Temperature

Superconductors-Materials

spects

Garmisch-Partenkirschen

W. Germany

Conference Secretariat: ICMC-

90-HTSC. Deutsche Gesel-

schaft für Metallkunde, eV.

Adenauerallee 21, 0-6370

Obenursel, W. Germany: (06171)

4081, fax (06171) 52554.

13-16

6ith Conference on Semi-

Insulating III-V Materials

Toronto, Canada

C. Miner, Bell-Northem Research

P. Box 3511 Station C Ottawa,

Ontario K1Y 4H7; tax (613)

763-2404

13-25

temational School on Crystal

Growth and Crystallographic

Assessment of Industrial

Materials 
21-25

CLEO/OEC-Conterence on Lasers and Electro-Optics Intemational Quantum Electronics Conterence

Anaheim, CA

Lasers and Electro-Optics Society 445 Hoes Lane, P.0. Box 1331, Piscataway, NJ 08855-1331; (201) 562-3895; tax (201) 562-1571

21-25

NETWORKS 90: 10th Polymer Networks Group Meeting and IUPAC 10th Intemational Symposium on Polymer Networks Jerusalem, Israel M. Gottlieb, Chemical Engineering Dept. Ben Gurion Univ., Beer Sheva 84105, Israel; 972-57461486; tax 972-57-31340

\section{3-25}

Fourth MiCon Symposium on Advances in Video Technology: Materials Science Applications San Francisco, CA ASTM, 1916 Race St., Philadel phia, PA 19103

2931

11th Intemational SAMPE European Chapter Conference Basil, Switzertand N. Hawley, 1055 W. San Bemardino Rd. Covina CA 91722; (818) 331-0616

$29-1$

E-MRS European Materials Research Society Meeting Strasbourg, France

P. Siffert, Centre de Recherches Nucléaires, Laboratoire PHASE, 67037 Strasbourg, Cedex

France; (88) 286543 ;

$\operatorname{tax}(88) 280990$

\section{JUNE 1990}

4-5

9th Annual Symposium on Electronics Materials, Processing. and Characterization

Richardson, TX

M.E. Weber, 9th Annual Symposium, P.O. Box 830914, Richarcson, TX 75083-0914; (214) 995-5983

4-6

Optical Spectroscopic Instrumentation and Techniques for the 1990s: Applications in Astronomy Chemistry, and Physics Las Cruces, NM

SPIE, P.O. Box 10, Bellingham, WA 98227-0010; (206) 676-3290; $\operatorname{tax}(206)$ 647-1445

4-8

Florida Advanced Materials Chemistry Conterence

Palmi Coast, FL

R.S. Drago, Dept. of Chemistry, Univ. of Florida, Gainesville, FL 32611
$5-7$

4th Intemational Conterence on Advanced Infrared Detectors and Systems

London, United Kingdom Institution of Electrical Engineers, Savoy Place, London WC2R OBL United Kingdom; 01-240 1871 ext. 222; tax 01-240 7735

6-8

High Performance Composites for the 1990s

Morristown, N

TMS, 420 Commonwealth Dr.,

Wamendale, PA 15086; (412) 776 9050; $\operatorname{tax}(412) 776-3770$

$10-13$

The Science Behind Materials

Synthesis

University Park, PA

K. Mourant, SMS Conference,

102 Materials Research Labora-

tory, Pennsylvania State Univer-

sity University Park, PA

16802-4801; (814) 865-1137

10-18

1st European East-West Symposium on Materials and Processes with High Industrial Potentia

Helsinkj, Finland

P. Attila, Tekonolink Oy, Hietalahdenkatu 2B, SF-00180 Helsinkj,

Findand; + 358-0-6801505; fax $+358-06801599$

$11-13$

5th Intemational Workshop on

Electroluminescence

Helsinlj, Finland

M. Leskelä, Dept. of Chemical

Engrg., Helsinbj Univ. of Tech.

SF-02150 Espoo, Finland; 358-0

451 2597; tax 358-0-462 373

11-14

4th Intemational Symposium on

Nondestructive Characterization

of Materials

Annapolis, MD

R.E. Green Jr., Johns Hopkins

University, Center for NDE,

Maryland Hall, Room 102,

Battimore, MD 21218; (301)

$338-7126$

$11-14$

11th Biennial Conference on

National Materials Policy

Williamsburg, VA

B. Houston, Federation of

Mateñals Societies, 1707 L St.

NW, Suite 333, Washington, DC

20036; (202) 296-9282

11.-15

Basic Course: SEM and X-Ray

Microanalysis

Bethtehem, PA

J.I. Goldstein, Dept. of Materials

Science and Engineering, Bldg. 5

Lehigh University, Bethlehem, PA

18015; (215) 758-5133
$11-15$

10th International Conterence on

Vacuum Metallurgy

Beijing, China

T. Shaojie, Chinese Society of

Metals, 46 Dongsixi Dajie, Beijing

100711, China; fax 5124122

12.14

4th Intemational Electronic

Materials and Processes

Conference

Albugquerque, NM

SAMPE, P.O. Box 2459, Covina,

CA 91722; (818) 331-0616;

$\operatorname{tax}(818)$ 332-8929

\section{3-15}

Atomic Layer Epitaxy

Helsinij, Finland

L. Nïniströ, Dept. of Chemical

Engrg. Helsinks Univ. of Tech.

Kemistintie 1, SF-02150 Espoo,

Finland; 358-0-4512600;

tax 3580-462373

$18-2$

Advanced Topics: SEM and X-Ray Microanalysis

Bethlehem, PA

J.l. Goldstein, Dept. of Materials

Science and Engineering, Bldg. 5 ,

Lehigh University, Bethlehem. PA

18015; (215) 758-5133

18-21

Analytical Electron Microscopy

Bethlehem, PA

J.I. Goldstein, Dept. of Materials

Science and Engineering, Bldg. 5,

Lehigh University, Bethlehem, PA

18015: (215) 758-5133

18-22

C-MRS Intemational ' 90

Beiling, China

Henge De Li, Dept. of Materials

Science \& Engineering, Tsinghua

Univ., Beifing 100084, China;

655646; tax (01) 2019583

(Soe related article in

(bl. XN, No. 9 )

21-22

Thin Specimen Preparation

Bethlehem, PA

J.I. Goldstein, Dept. of Materials

Science and Engineering, Bldg. 5,

Lehigh University, Betthlehem, PA

18015; (215) 758-5133

24-30

7th CIMTEC - Whild Ceramics

Congress

Florence, Italy

Seventh CIMTEC, P.O. Box 174,

48018 Faenza, Italy; 546-664143;

$\operatorname{tax} 546-664138$

26-28

Computer-Aided Assessment and

Control of Localized Damage

Ashurst Southampton, United

Kingdom

J. Futers, Computationa

Mechanics Institute, Ashurst

Lodge, Ashurst, Southampton

SO4 2AA, United Kingdom:

(042129) 3223; (042129) 2853
27.29

Electronic Materials Conference

Santa Barbara, CA

VG. Keramides, Bellcore, 331

Newman Springs Rd., Red Bank

NJ 07701; (201) 758-3353; tax

(201) $758-9627$

\section{JULY 1990}

2-5

7th CIMTEC Satellite Symposium

4. High-T, Superconductors

(See June 24-30 CIMTEC listing)

Trieste, Italy

7th CIMTEC, Satellite Symposium

4. P. . Box 174, 48018

Faenza, Italy; 546-664143;

$\operatorname{tax} 546-664138$

8-13

International Symposium on

Optical and Optoelectronic

Applied Science and Engineering

San Diego, CA

SPIE P. B BOX 10 , Bellingham,

WA 98227-0010; (206) 676-3290;

$\operatorname{tax}(206) 647-1445$

Conference on Nondestructive

Evaluation of Modem Ceramics

Columbus, $\mathrm{OH}$

American Ceramic Society, 757

Brooksedge Plaza Drive, Wester

ville, OH 43081; (614) 890-4700

$\operatorname{tax}(614) 8996109$

8th American Conterence on

Crystal Growth

Vail, $C O$

B.L. Sopori, Solar Energ

Research Institute, 1617 Cole

Bivd., Golden, CO 80401-3393;

(303) 231-1383

$17-19$

Low Temperature Engineering and

Cryogenics Conference, LTEC'90

Southampton, England

Cryogenics Conference Office.

Institute of Crogenics, University

of Southampton, $\$ 095 \mathrm{NU}$

United Kingdom; 0703-595000,

ext. 2059 or 2662 ; tax 0703 .

593939

22-27

3 th Intemational Liquid Crysta

Conference

Vancouver, Canada

B. Bergersen, Dept. of Physics,

Univ. of British Columbia

Vancouver, V6T 2A6 Canada;

(604) 228-2603

$22-3$

NATO Advanced Study Institute on Diamond and Diamond-Like Films

Castelvecchio Pascoli, Italy

C.J. McHargue, Metals and

Ceramics Div., Oak Ridge National

Lab PO Box 2008 , Oak Ridge

TN 37831-6118; (615) 574-4344

$\operatorname{tax}(615) 5747659$

23-26

Conference on Advances in

Cementitious Materials

Gaithersburg, MD

American Ceramic Society, 757

Brooksedge Plaza Drive, Wester-

ville, OH 43081; (614) 890-4700

$\operatorname{tax}(614) 8996109$ 
$31-2$

4th Intemational Conterence on Shallow Impurities in Semiconductors

London, United Kingdom

G. Davies, Physics Department,

Kings College London, The

Strand, London WC2R 2LS

United Kingdom

\section{AUGUST 1990}

8-10

Specialty Polymers ' 90

Battimore, MD

W. Harvey, SP '90, Butterworth

Scientific Ltd. P.O. Box 63

Westbury House, Bury St.,

Guildford, Surrey, GU2 5BH,

United Kingdom; 0483 300966.

tax 0483301563

12-18

12th Intemational Congress for

Electron Microscopy

Seattle, WA

R.M. Fisher, Univ. of Washington,

5001 25th Ave. NE, GH-22

Seattle, WA 98195; tax (206)

545-9359

13-15

LT-19 Satellite Conference on High

Temperature Superconductivity

Cambridge, United Kingdom

K.S. Diffey, IRC in Super-

conductivity, Madingley Rd.

Cambridge CB3 OHE, UK; (0223)

337472; $\operatorname{tax}(0223) 337074$

13-17

7th International Conference on

Rapidly Quenched Materials

Stockholm, Sweden

Dept. of Casting of Metals, Royal

Institute of Technology, S-100 44

Stockholm, Sweden; + 46-8-790

79 84; $\operatorname{tax}$ + 46-8-1091 99

16-21

Intemational Conference-

Chemistry of Electronic Ceramic

Materials

Targhee National Forest, WY

R.S. Roth, NIST, Ceramics Div.

420, Bidg. 223, Rm. B216

Gaithersburg, MD 20899; (301)

975-6116; fax (301) 975-2128

26-31

Light. X-Ray and Neutron

Scattering and Reflectivity from

Polymers: American Chemical

Society National Meeting

Washington, D.C.

P.M. Cotts, K93/802, IBM

Almaden Research Ctr. 650

Harry Rd., San Jose, CA 95120

6099; (408) $927-1638$

27-30

10th International Symposium on

Boron, Borides, and Related

Compounds

Albuquerque, NM

T.L. Aselage, Div. 1842, Sandia

National Labs. Albuquerque, NM

87185-5800; (505) 844-0949
27-31

6th Thieste Semiconductor

Symposium: Hydrogen in Semi-

conductors

Trieste, Italy

M. Stutzmann, Max Planck

Institut fur Festkopertorschung

Heisenbergstrasse 1, 0-7000

Stuttgart 80, W. Germany;

(0711)68 60-1; $\operatorname{tax}(0711) 6874371$

27-31

6th Intemational Conference on

Molecular Beam Epitaxy

LaJolla, CA

CW. Tu, Dept of Elect and

Comp. Engineering, MC R-007,

Univ. of Califf., San Diego, La

Jolla, CA 92093-0407; (619) 534

4687; $\operatorname{tax}(619)$ 534-2486

$28-31$

International Conterence on

Computer Applications to Materials

Science and Engineering

Tokyo, Japan

M. Doyama, CAMSE '90, Nikkan

Kogyo Shimbun Ltd. Business

Bureau, 8-10 Kudan Kita, 1 -

chome, Chiyoda-ku, Tokyo 102,

Japan; (03) 222-7162; $\operatorname{tax}(03)$

221-7137

\section{SEPTEMBER 1990}

37

6th Europhysics Top Conference:

Lattice Defects in lonic Materials

Groningen, The Netherlands

H.W. den Hartog, Solid Physics

Lab, Melkweg 1, NL-9718 EP

Groningen

5-7

Analysis by a Combination of lon Beam (Accelerator-Based) and

Surface Specific Techniques

Namur, Belgium

G. Demortier, Département de

Physique, Facultes Universitaires

N-D de la Paix, ne de Bnuxelles,

61. B-5000 Namur, Belgium; tax 32-81-2303 91

$9-14$

MIRISIIon Beam Modficication of

Matertals_-1BMM ' 90

Knoxville, TN

P.H. Green, IBMM '90, Oak Ridge

National Lab. Solid State Div.

P.0. Box 2008, M.S.6033, Oak

Ridge, TN 37831-6033;

(615) $576-1864 ; \operatorname{tax}(615)$

$574-4143$

(See related article in

Vol. XV No. 12.)

10-13

2nd World Congress on Super-

conductivity

Houston, TX

World Congress on Super-

conductivity, P.0. Box 27805

Houston, TX 77227-7805; (713)

895-2500; $\operatorname{tax}(713)$ 623-3560

$10-14$

2nd international Conterence on

Plasma Surface Engineering

Garmisch-Partenkirchen, W.

Germany Deutsche Gesellschaft

für Galvano-u. Oberfächentech-

nike. V. Horionplatz 6, 0-4000

Dusseldorf 1, W. Germany; 0211/

132381
$10-20$

NATO Advanced Study Institute

on Applications of Metallic and

Ceramic Superconductivity

Fort Collins, $\mathrm{CO}$

H. Weinstock, AFOSR/NE, Bolting

AFB Washington, DC 20332

6448; (202) 767-4933; $\operatorname{tax}(202)$ $767-0466$

11-12

Workshop on High-Density

Plasma Techniques and Proc-

esses for Integrated Circuit

Fabrication

Burfingame, CA

Continuing Education in Engineer-

ing, UC Berkeley Extension, 2223

Fulton St., Berkeley, CA 94720;

(415) 642-4151; tax (415)

$643-8683$

$11-13$

3nd International SAMPE Metals

Conference

Williamsburg, VA

N. Hawley, 1055 W. San Bernar-

dino Rd. Covina, CA 91722

(818) $331-0616$

$17-19$

MIRS 2nd Intemational

Conterence on Electronits

Materials (ICEM-II)

Hoboken, NJ

J. Stokes, Materials Research

Society, 9800 McKnight Rd.

Pittsburgh, PA 15237; (412) 367 -

3003; tax (412) 367-4373

(See related article in

Vol. XVN No. 12.)

$17-20$

Applied Optics and Opto-

Electronics

University of Nottingham, United

Kingdom

Applied Optics Group, Blacket

Laboratory Imperial College.

London SW7 2BZ; (01) 5895111

ext. 6975/6842

18-20

Microcircuit Engineering '90

Leuven, Belgjum

K. Legein, IMEC vow, Kapeldreef

75. B-3030, Leuven, Belgium

23-27

MIRIS 2nd Intemational

Conference on New Diamond

Sclences and Technology

Crystal City, VA

R. Messier, Engineering Science

and Mechanics, Pennsylvania

State Univ., 265 Materials

Research Lab. University Park,

PA 16802; (814) 865-3704; fax

(814) 863-7039

(See related article in this issue.)

24-27

European Gallium Arsenide

Conterence

St. Helier, Jersey, Channel islands

Meetings Officer, Institute of

Physics, 47 Belgrade Square,

London SW1X 80X, England

24-28

1990 Applied Superconductivity

Conterence

Aspen, $\mathrm{CO}$

ASC/90, Centennial Conferences,

5353 Manhattan Circle, Suite 103

Boulder, CO 80303; (303)

499-2299

\section{OCTOBER 1990}

36

American Society of Composites:

Fourth Technical Conterence on

Composite Materials

Blacksburg, VA

M.W. Hyer, RSM Dept., Virginia

Tech., Blacksburg, VA 24061;

(703) 231-5372

$7-11$

TMS Fall Meeting: Physical

Metallurgy and Materials

Defroit, MI

TMS, 420 Commonwealth Dr.

Wamendale, PA 15086; (412) 776

9050; $\operatorname{tax}(412) 776-3770$

8-12

37th National Vacuum

Symposium

Toronto, Ontario, Canada

M. Churchiil, American Vacuum

Society, 335E. 45th St., New

Yonk NY 10017: (212) 661-9404

$14-19$

178th Meeting of The Electro-

chemical Society

Seattle, WA

The Electrochemical Society, 10

S. Main St. Pennington, NJ

085342896

15-17

ISHM '90: Intemational Sympo-

sium on Microelectronics

Chicago, IL

N. Binkley, ISHM, P.O. Box 2698

Reston, VA 22090-2698; (703)

471-0066; tax (703) 471-1937

21-24

11 th International Workshop on

Rare-Earth Magnets and Their

Applications

Pittsburgh, PA

S.G. Sankar, Camegie Mellon

University, Mellon Institute, $\mathbf{4 4 0 0}$

Fifth Ave., Pittsburgh, PA 15213;

(412) 268-5649; tax (412) 2683101

6th Intemational Symposium on

Magnetic Anisotropy and

Coencivity in Rare-Earth-Transition

Metal Alloys

Pittsburgh, PA

S.G. Sankar, Camegie Mellon

University Mellon Institute, 4400

Fifth Ave., Pittsburgh, PA 15213;

(412) 268-5649; $\operatorname{tax}$ (412)

68.3101

27.2

Intemational Conference on Physical Concepts of Materials tor Novel Optoelectronic Device 